\title{
A Unified Framework for $k$-Coverage and Data Collection in Heterogeneous Wireless Sensor Networks
}

\author{
Habib M. Ammari \\ Wireless Sensor and Mobile Ad-hoc Networks (WiSeMAN) Research Lab \\ Department of Computer and Information Science, College of Engineering and Computer Science \\ University of Michigan-Dearborn, Dearborn, MI 48128, USA \\ hammari@umd.umich.edu
}

\begin{abstract}
One of the fundamental tasks in the development of wireless sensor networks is coverage, which measures the network effectiveness and accuracy in event detection. While most existing studies on coverage focus on homogeneous and static wireless sensor networks, where the sensors have the same features, such as sensing, communication, and initial energy reserve, this paper considers heterogeneous sensors and sink mobility, which provide a more realistic and accurate view of the network design for a variety of sensing applications. In this paper, we exploit Helly's Theorem to address the joint problem of $k$-coverage and data collection in heterogeneous wireless sensor networks, where each point in a field of interest is simultaneously covered by at least $k$ active heterogeneous sensors. More precisely, we introduce a global framework that jointly considers $k$-coverage and data collection. Precisely, we propose a multi-tier (or hierarchical) architecture of heterogeneous sensors along with two data collection protocols. While the first protocol is based on an adaptive hybrid forwarding scheme, the second one uses a mobile sink to collect the sensed data from all the sensors in the network. To this end, we investigate the optimal mobility strategy of the sink in order to minimize the average total energy consumption due to both of data communication and sink mobility in a circular sensor field. We divide the field into concentric circular bands with the same width, and derive a closed-form solution for the optimal sink mobility. We corroborate our analysis with simulation results to assess our proposed framework. We find that our sink mobilitybased data collection protocol outperforms our hybrid geographic forwarding-based data collection protocol.
\end{abstract}

Keywords: Wireless sensor nets, $k$-coverage, data collection, heterogeneity, hierarchical deployment, adaptive forwarding, sink mobility, Helly's theorem.

\section{Introduction}

A wireless sensor network (or simply sensor net) consists of tiny, low-powered sensors that communicate with each other (possibly) in a multi-hop fashion and report their sensed data to a central gathering component, called the sink, for further analysis and processing. Sensor nets can be used in a wide variety of civilian, environmental, natural, and military applications, such as health monitoring, environmental monitoring, seism monitoring, and battlefields surveillance, respectively. The design of sensor nets faces a major problem due to the very scarce resources of the sensors, such as battery power (or energy), bandwidth, CPU, and storage, to name a few, with energy being the most critical one.

Coverage and connectivity have been always considered among the major research problems in sensor nets [31]. While coverage informs how well a field is monitored, connectivity is concerned with how well sensors in a network communicate with each other. A more general concept of coverage is called $k$-coverage, where each point in a field is covered (or sensed) by at least $k$ sensors simultaneously (or simply $k$-covered). The problem of joint $k$-coverage and data collection in sensor nets is one of the fundamental research problems in sensor nets. An efficient solution to the problem of $k$-coverage in sensor nets consists of finding a minimum subset of sensors $S$ such that each point in a field is $k$-covered by sensors in $S$. Although the connected $k$-coverage problem in sensor nets has been studied extensively in the literature [5], [12], [13], most existing work focused on homogeneous sensor nets, where all the sensors have the same capabilities with regards to their storage, computational power, sensing range, communication range, and initial energy, to name a few. Also, the problem of $k$-coverage-preserving scheduling (or sensor duty-cycling) in homogeneous sensor nets has gained considerable attention [1], [24], [26], [31], [32]. However, this type of homogeneous sensors poses a severe restriction on the design of real-world sensor net applications given that all the sensors are required to have the same above-mentioned capabilities. In general, this assumption is unrealistic as the sensors may not necessarily have the same capabilities even when they are built by the same company. In other words, sensors may be heterogeneous.

\subsection{Motivations}

Our work is motivated by the following observations. First, in real-world applications, sensor nets have heterogeneous sensors which do not necessarily have the same capabilities (i.e., sensing range, communication range, energy, storage, computation, etc.). These networks have a potential to increase the network lifetime and reliability without causing significant increase in their cost [28]. For instance, Intel deployed two types of sensors (line-powered and batterypower sensors) in the design of a pilot application of sensor nets in order to monitor the health of mechanical equipment in its fabrication plants [28]. While line-powered sensors can be attached to pumps and motors in the fabrication plant, 
battery-power sensors can be used to reduce installation cost and complexity. Indeed, Yarvis et al. [28] presented several analytical, and testbed results showing the potential benefit and impact of energy and heterogeneity on sensor nets, where all the sensors report their data to a sink. Second, the design of sensor nets for planet exploration [22], multiplesensor data fusion [15], and triangulation-based positioning systems [21], require degree of coverage $k \geq 3$. Third, the use of static sink potentially yields the problem of energy sinkhole, where the sensors around the sink are continuously involved in forwarding data on behalf of all other sensors to the sink. This causes a severe problem of depletion of their battery power (or energy), thus, isolating the sink. To remedy this problem, we use a mobile sink for data collection.

\subsection{Problem Formulation}

In this paper, we consider heterogeneous sensors deployed in a field of interest. These sensors may differ by their sensing range, communication range, and/or energy reserve. More specifically, we focus on the problem of joint $k$-coverage and data collection in heterogeneous sensor nets using a single mobile sink. This problem can be stated as follows:

Given a deployment field and a set $S$ of heterogeneous sensors, we want to address the following questions:

- How to select a minimum subset of sensors $S^{\prime} \in S$ to stay active, and how to place them in the field so that each point in the field is $k$-covered, where $k \geq 3$ is the degree coverage needed by the sensing application?

- What is the optimal mobility trajectory of the sink so as to minimize the average total energy consumption due to data communication and sink mobility?

- How can data be collected efficiently in this type of duty-cycled $k$-covered sensor net?

It is worth mentioning that the problem of selecting a minimum subset of sensors to $k$-cover a field using homogeneous sensors is NP-hard [12]. With heterogeneous sensors, this problem is also NP-hard. Intuitively, the NPhardness in the homogeneous case leads to the NP-hardness in the heterogeneous case. Thus, we propose approximation algorithms to solve the joint $k$-coverage and data collection problem in heterogeneous sensor nets with one mobile sink.

\subsection{Major Contributions}

Our contributions in this paper can be described as follows:

- First, we propose a general framework for $k$-coverage and mobile data collection in sensor nets using a single mobile sink. This framework accounts for heterogeneous sensors, which do not have the same sensing range, communication range, and initial energy. Precisely, we propose centralized and distributed protocols for generating energy-efficient $k$-coverage configurations in heterogeneous sensor nets and data collection via a mobile sink. Precisely, we suggest a pseudo-random (i.e., not fully random) sensor deployment approach, where the sensors are deployed in different layers in a circular sensor field according to their strengths with regard to their sensing range, communication range, and initial energy. Then, we propose energy-efficient centralized and distributed $k$-coverage protocols based on this pseudo-random deployment approach.

- Second, we suggest a data collection protocol based on an adaptive hybrid forwarding scheme, where the sensors could adaptively choose between short-range and long-range forwarding based on their location with respect to the sink. Moreover, as it is hybrid, the sensors take advantage of deterministic and opportunistic forwarding by specifying in their sensed data packets the id's of $m$ active candidate next forwarders from their communication neighbor set, where $m>1$.

- Third, we investigate the optimal mobility strategy of the sink during data collection with a goal to minimize the average total energy consumption due to data transmission and reception as well as sink mobility in a circular sensor field. To this end, we divide the field into concentric circular bands with the same width, and derive a closed-form solution for the optimal sink mobility. We use this analysis to design a data collection protocol using a mobile sink on top of our multi-tier sensor deployment architecture. To the best of our knowledge, this paper provides the first analysis of the best mobility trajectory of the sink during data collection in heterogeneous $k$-covered sensor nets.

- Fourth, we corroborate our analysis with simulation results to assess the performance of our framework. We find that our sink mobility-based data collection protocol outperforms our adaptive hybrid geographic forwardingbased data collection protocol. In addition, we find that our pseudo-random sensor deployment outperforms random sensor deployment to ensure $k$-coverage. Also, we find that our sink mobility-based data collection protocol outperforms our hybrid geographic forwardingbased data collection protocol in terms of average delay and data delivery ratio.

\subsection{Paper Organization}

The remainder of this paper is organized as follows. Section 2 presents some key definitions and widely used assumptions for $k$-coverage in sensor nets. Also, it introduces the energy model that we used in our analysis of the $k$-coverage problem in heterogeneous sensor nets. Section 3 reviews a sample related work on coverage in both of homogeneous and heterogeneous sensor nets. Section 4 addresses the problem of $k$-coverage in heterogeneous sensor nets. Section 5 discusses our adaptive hybrid geographic forwarding-based data collection protocol, while Section 6 shows our analysis of the optimal sink mobility trajectory for data collection in heterogeneous $k$-covered sensor nets. Section 7 evaluates the performance of our proposed framework for joint $k$-coverage and data collection. Section 8 concludes the paper. 


\section{Related Work}

In this section, we review a sample approaches for coverage and connectivity in sensor nets. A special interest is in heterogeneous sensor nets.

The problem of coverage and connectivity in sensor nets was originally addressed by Xing et al. [26]. They proposed the CCP protocol that provides different degrees of full coverage of a convex region [26]. In [1], we showed that our proposed $k$-coverage protocol using homogeneous sensors requires less active sensors than $\mathrm{CCP}$ to achieve the same degree of coverage, thus yielding significant energy savings. Yang et al. [27] formalized the $k$-connected coverage set problem in terms of linear programming and proposed a nonglobal solution to it. Also, Nakamura et al. [20] provided a linear programming formulation of the coverage problem. Chen et al. [9] proposed a protocol, called span, which chooses which nodes to stay active based on their energy. Likewise, Vieira et al. [23] utilized the area of the Voronoi cell to determine which nodes to stay awake. In [13], Huang et al. studied the relationship between coverage and connectivity of sensor nets and proposed distributed protocols to ensure both of them. Bai et al. [5] proposed an optimal deployment strategy for coverage and 2-connectivity regardless of the relationship between sensing and communication radii of the sensors. Gupta et al. [12] proposed centralized and distributed algorithms for connected sensor cover so the sensor net selforganizes its topology in response to a query and activate the necessary sensors to process the query. Zhang and Hou [31] proposed an optimal geographical density control protocol to keep a small number of sensors active regardless of the ratio of the communication range to the sensing range of sensors.

While coverage and connectivity in homogeneous sensor nets have been studied well, their study and analysis in heterogeneous sensor nets have received little attention. Ammari et al. [2] proposed a multi-tier architecture of heterogeneous sensors to better address the problems introduced by pure randomness and heterogeneity in sensor nets. The work presented in this paper is an extension of the one published in [2]. While the work in [2] focuses on the problem of $k$-coverage, this paper tackles the problem of joint $k$-coverage and data collection in sensor nets in both static and mobile sensor nets that has only one mobile sink. Wang et al. [25] proposed a fine analysis of coverage using two types of sensors with different capabilities and discussed the impact of heterogeneous sensing and communication ranges of the sensors on coverage and broadcast reachability. Duarte-Melo and Liu [11] focused on heterogeneous sensors equipped with different battery power in their analysis of a clustering approach while considering two sensor deployment strategies. Lazos et al. [16] addressed the problem of detecting mobile targets using sensors that have heterogeneous sensing areas of arbitrary shape with a goal to increase the robustness of target detection. Specifically, they considered a deterministic sensor deployment strategy to maximize the probability of target detection. Ma et al. [19] proposed a resource oriented protocol that implements a network topology according to the number of heterogeneous sensors as well as their specific resources and characteristics, such as radio coverage, power capacity, processing capabilities, and mobility attributes. Du and Lin [10] proposed a differentiated coverage algorithm for heterogeneous sensor nets. The motivation behind providing different degrees of sensing coverage for different regions is that different network areas do not have the same importance and hence some areas require higher coverage degree than others. Yuce et al. [30] proposed a heterogeneous sensor network system for monitoring physiological parameters from multiple patient bodies. Their system provides patients with mobility, and medical staff with flexibility in obtaining physiological data on the patients on-demand through the Internet. Mhatre et al. [18] considered a heterogeneous sensor net with two types of nodes which differ by their intensity $\lambda$ (or average number per unit area) $\left(\lambda_{0}, \lambda_{1}\right)$ and their battery energy $\left(E_{0}, E_{1}\right)$. While type 1 nodes perform sensing, type 2 nodes perform sensing and act as cluster heads. Their study consists of computing the optimum node intensities $\left(\lambda_{0}, \lambda_{1}\right)$ and node energies $\left(E_{0}, E_{1}\right)$ that ensure connected coverage of the surveillance area with a high probability while guaranteeing a network lifetime of at least $T$ units and minimizing the overall cost of the network.

\section{Preliminaries}

In this section, we present some definitions and widely used assumptions for $k$-coverage in sensor nets. Also, we describe the energy model for our analysis of the above problem.

\subsection{Definitions}

Definition 1: The sensing neighbor set of a sensor $s_{i}$ consists of all sensors in the sensing range of $s_{i}$.

Definition 2: The communication neighbor set of a sensor $s_{i}$, denoted by $N S\left(s_{i}\right)$, includes all sensors located in the communication range of $s_{i}$.

Definition 3: A point in a field is said to be $k$-covered if it belongs to the intersection of the sensing disks of at least $k$ sensors. A region $A$ is said to be $k$-covered if every point in $A$ is $k$-covered. A $k$-covered sensor net is a network that $k$ covers a field. We call coverage degree provided by a sensor net the maximum value of $k$ such that a field is $k$-covered.

Definition 4: A sensor $s_{i}$ can communicate with another sensor $s_{j}$ if $s_{j}$ is in the communication neighbor set of $s_{i}$ or there is at least one communication path $s_{i}, \ldots, s_{l}, s_{l+1}, s_{l+2}$, $\ldots, s_{j}$ such that $s_{l+1}$ belongs to the communication neighbor set of $s_{l}$, for $i \leq l \leq j-1$. A sensor net is said to be connected if a sensor $s_{i}$ can communicate with any other sensor $s_{j}$ in the network.

Definition 5: The width of closed convex area $A$ is the maximum distance between any pair of parallel lines that bound $A$. 


\subsection{Assumptions}

Assumption 1: All the sensors are static and aware of their locations via a localization technique [8].

Assumption 2: All the sensors are randomly and uniformly deployed in a circular field unless stated otherwise.

Assumption 3: All the sensors are supposed to be heterogeneous, i.e., do not have the same sensing range, communication range, and initial energy.

Assumption 4: The sensing and communication ranges of a sensor $s_{i}$ are represented by disks of radii $r_{i}$ and $R_{i}$, respectively.

Assumption 5: We consider a deterministic sensing model, where a point $p$ in a field is covered by a sensor $s_{i}$ if $\delta\left(p, s_{i}\right) \leq$ $r_{i}$, where $\delta\left(p, s_{i}\right)$ stands for the Euclidean distance between $p$ and $s_{i}$.

\subsection{Energy Consumption Model}

We assume that the energy consumption of the sensors is due to data reception, transmission, and mobility. According to [12], the energy spent in transmitting one message of size $\kappa$ bits over a distance $d$, is given by

$$
E_{t x}(d)=\kappa \varepsilon d^{\alpha}+\kappa E_{\text {elec }}=\kappa\left(\varepsilon d^{\alpha}+E_{\text {elec }}\right)
$$

where $E_{\text {elec }}=50 \times 10^{-9}$ is the electronics energy, $\varepsilon$ is the transmitter amplifier $\left(\varepsilon=10^{-11}\right.$ for $\alpha=2$, and $\varepsilon=13 \times$ $10^{-16}$ for $\alpha \geq 3$ ), and $\alpha$ is the path-loss exponent ( $2 \leq \alpha \leq$ 4) [12]. Also, the energy spent in receiving one $\kappa$-bit message is given by:

$$
E_{r x}=\kappa E_{\text {elec }}
$$

Hence, the total energy consumed when a sensor $s_{i}$ transmits a $\kappa$-bit message over a distance $d$ to $s_{j}$, and $s_{i}$ receives it, is

$$
E_{\text {trx }}(d)=\kappa\left(\varepsilon d^{\alpha}+2 E_{\text {elec }}\right)
$$

Following [25], the energy spent due to mobility, denoted by $E_{m o b}\left(d_{m o b}\right)$, is computed as

$$
E_{m o b}\left(d_{m o b}\right)=e_{m o v e} d_{m o b}
$$

where $e_{\text {move }}$ stands for the energy cost for a mobile sensor to move one unit distance, and $d_{m o b}$ is the total distance traveled by the mobile sensor. Without loss of generality, we assume that $\kappa=256$ and $\alpha=2$.

\section{Heterogeneous $k$-Coverage}

In general, sensor nets may have various types of sensors, thus, increasing the network reliability and lifetime [28]. In this section, we focus on heterogeneous $k$-covered sensor nets to generate energy-efficient $k$-coverage configurations. To this end, we exploit our previous results on $k$-coverage for homogeneous sensor nets [1] based on Helly's Theorem [7]. We will extend those results to account for the case of heterogeneous sensors deployment.

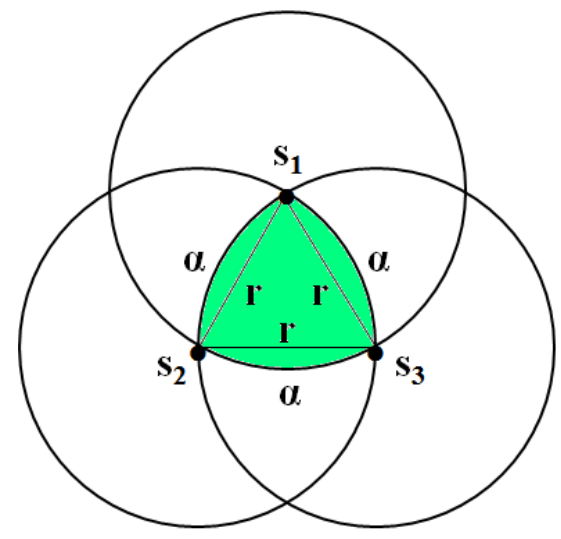

Figure 1: Reuleaux triangle

\subsection{Previous Results}

For the sake of self-containment and completeness, we provide a brief overview of our previous results with regard to the problem of $k$-coverage in homogeneous sensor nets, without their proofs. For more details about the proofs of these results, the interested reader is referred to our earlier work published in [1]. First, we state Helly's powerful theorem of convexity theory [7].

Helly's Theorem [7]: If the intersection of any $m$ convex sets of $E$ in $R^{n}$ is not empty, then, the intersection of all convex sets of $E$ is not empty, where $m \geq n+1$.

Lemma 1 [1] is an instance of Helly's Theorem [7] in a two-dimensional space that characterizes the intersection of $k$ sensing disks, with $n=2$ and $k=m$.

Lemma 1 [1]: Let $k \geq 3$. The intersection of $k$ sensing disks is not empty if the intersection of any three of those $k$ sensing disks is not empty.

Based on Lemma 1, Lemma 2 [1] gives a sufficient condition for $k$-coverage of a two-dimensional field.

Lemma 2 [1]: Let $k \geq 3$. A two-dimensional field is $k$ covered if any Reuleaux triangle [33] of width $r$ (or simply slice) in the field contains at least $k$ active sensors, where $r$ is the radius of the sensors' sensing disks.

Notice that a Reuleaux triangle [33] (Figure 1) can be obtained when three sensors, say $s_{1}, s_{2}$, and $s_{3}$, are symmetrically located from each other so that the distance between any pair of sensors is equal to $r$. It has a regular triangle of side length $r$ with a curved region on each side. Also, a Reuleaux triangle has a constant width equal to $r$. Notice that the sensing disk of a sensor can be covered by six overlapping slices of width $r$. Thus, the minimum overlap area of two adjacent slices forms a lens, as shown in Figure 2. Lemma 3 characterizes $k$-coverage, while Theorem 1 states a tight sufficient condition for $k$-coverage of a twodimensional field of interest based on Lemma 3.

Lemma 3 [1]: $k$ active sensors located in the lens of two adjacent slices can $k$-cover both slices. 


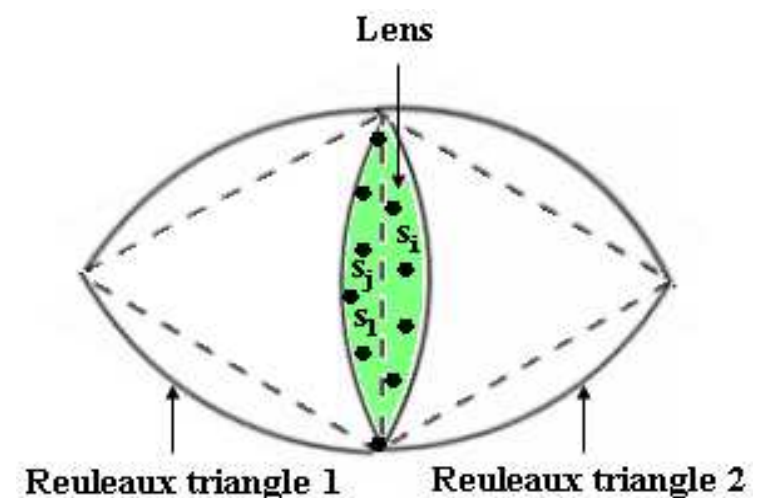

Figure 2: Lens

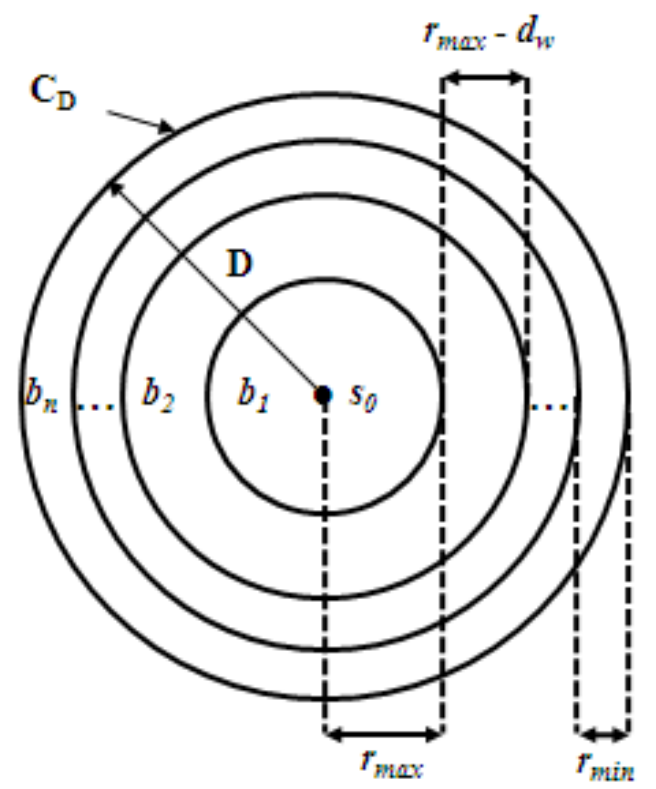

Figure 3: A field decomposed into circular bands

Theorem 1 [1]: Let $k \geq 3$. A field is $k$-covered if for any slice of width $r$ in the field there is another adjacent slice of width $r$ such that their lens contains at least $k$ sensors.

Next, we propose an efficient deployment strategy, where the sensors are pseudo-randomly deployed in the field, in order to exploit the benefits of heterogeneity.

\subsection{Pseudo-Random Deployment}

We consider a circular field and heterogeneous sensors that have different radii of sensing and communication ranges, and initial energy. It is well known that in any static sensor net, the sensors located nearer a static sink are heavily used in forwarding data to it, thus suffering from severe energy depletion, known as sink hole problem, which we have addressed in [3]. Precisely, we investigated a variety of mobility scenarios of a single or several mobile proxy sinks, as well as various placement strategies of one or multiple static sinks in a two-dimensional deployment field during data collection. In this paper, in order to cope entirely with the sink hole problem, we adopt a different approach that jointly uses sensor heterogeneity and sink mobility. Precisely, we propose to deploy heterogeneous sensors, where the most powerful sensors are located nearer the sink. Furthermore, we use a single mobile sink. It is important to design energy-efficient $k$-coverage protocols that help extend the network lifetime when sensed data routing is considered. In other words, these protocols should be designed in a way to facilitate the development of a uniform framework that jointly considers sensor duty-cycling for $k$-coverage, and data forwarding on duty-cycled sensors. In fact, the ultimate goal of the design of sensor nets is to monitor an environment and generate sensed data to be forwarded to the sink for further analysis and processing.

Assume that the minimum and maximum radii of the sensing range of the sensors are $r_{\min }$ and $r_{\max }$, respectively, and that the radius of the circular field is $D$. We propose to slice this circular field, denoted by $C_{D}$, into $n$ concentric circular bands of strictly decreasing widths starting from the inmost band. Each band has homogeneous sensors. But, any two bands in the field have two different sets of sensors. More specifically, the sensors located in the band closer to the center of the circular field are more powerful than the sensors in the other band. Also, we assume that the difference in width between any two adjacent bands is $d_{w}$. More specifically, the inmost band, denoted by $b_{1}$, has a width equal to $r_{\max }$ while the outmost band, denoted by $b_{n}$, has a width equal to $r_{\min }$ as shown in Figure 3.

The motivation behind decomposing a field into bands with such widths is the fundamental result stated by Xing $e t$ $a l$. [26]. They proved that a sensor net is connected when the radius of the communication range of the sensors is at least equal to twice the radius of their sensing range provided that coverage is guaranteed. In our sensor deployment strategy, the width $w_{j}$ of a given band $b_{j}$ is computed such that the radius $R_{j}$ of the communication range of any sensor $s_{j}$ placed in $b_{j}$ should be at least equal to the sum of the width of $b_{j}$ and its adjacent predecessor band $b_{j-1}$. That is,

$$
R_{j} \geq w_{j}+w_{j-1}
$$

where $j \geq 2, w_{j}=r_{j}$ and $w_{j-1}=r_{j-1}=r_{j}+d_{w}$, with $r_{j-1}$ being the radius of the sensing range of any sensor $s_{j-1}$ located in band $b_{j-1}$ and $d_{w}$ is the difference in width between bands $b_{j-1}$ and $b_{j}$ as discussed earlier. We should recall that the width of the inmost band $b_{1}$ is $r_{\text {max }}$ and that of the outmost band $b_{n}$ is $r_{\text {min }}$. Figure 3 illustrates this discussion. This design ensures network connectivity to enable communication between the sensors and facilitate forwarding of the sensed data generated by the sensors toward the sink. This issue becomes clearer when a geographic forwarding protocol is built on top of our connected $k$-coverage protocols, where all the sensors participating in the $k$-coverage form a connected network. From now on, we use the terms "connected $k$-coverage" and " $k$-coverage" interchangeably. Our sensor deployment approach is hierarchical due to the presence of multiple bands forming the sensor field. Also, it is pseudo-random in 
the sense that the sensors are deployed densely and randomly within each band while ensuring that the sensors in any pair of bands in the circular field are heterogeneous. Our heterogeneous sensor deployment approach is designed in a way that all the sensors placed in the band $b_{j}$ are homogeneous, and, thus, have the same sensing range whose radius $r_{j}$ is computed as follows:

$$
r_{j}=r_{\max }-(j-1) \times d_{w}
$$

where $d_{w}>0$ and $r_{\max }>r_{\min }$.

Our deployment strategy satisfies the following equations, where $n$ stands for the number of bands (i.e., number of types of sensors) forming the circular field $C_{D}$ :

$$
\begin{aligned}
& r_{\text {min }}+(n-1) \times d_{w}=r_{\text {max }} \\
& n \times r_{\text {min }}+n \times(n-1) \times d_{w} / 2=D
\end{aligned}
$$

with $n$ and $d_{w}$ being the only unknowns in the above equations. This is a valid assumption since we know only the capabilities of the sensors in terms of their sensing range, namely $r_{\max }$ and $r_{\min }$, and the radius of the circular field $D$. Therefore, knowing $r_{\max }, r_{\min }$, and $D$, it is easy to check that there is a unique solution $\left(n, d_{w}\right)$ to the above equations. For instance, assume that we have $r_{\min }=1, r_{\max }=3$, and $D=16$, we find that $n=8$ (i.e., there are 8 bands) and $d_{w}=2 / 7$. Next, we describe in details both of our centralized and distributed connected $k$-coverage configuration protocols which exploit the characteristics of the sensors in their respective bands.

\subsubsection{Centralized $\boldsymbol{k}$-Coverage Protocol}

Generally, the sink is attached to an infinite source of energy, such as a wall outlet, and, thus, has no energy limitation. Thus, the single-point failure problem does not exist when the sink plays any particular role as the battery depletion problem for the sink cannot arise. In our centralized protocol (PR-Het-CCC ${ }_{k}$ ), we assume that the sink is responsible for randomly decomposing each of these bands into overlapping Reuleaux triangles ("PR" stands for "pseudo-random"). This implies that the sink is aware of the locations of all the sensors. Also, we assume that every sensor knows the $i d$ of the band it belongs to. Then, the sink applies the result of Theorem 1 to select a subset of sensors to $k$-cover each of these bands with the necessary number of sensors. The sink will have to send a request to each band, where it specifies a band $i d$ and the $i d s$ of a subset of sensors located in that band so they remain active to $k$-cover the underlying band. We assume that each sensor is uniquely identified by a natural number $i d$.

The sink performs these actions at the beginning of each round under the assumption that all the sensors are awake at the beginning of a round and during some time interval $t_{\text {awake }}$. This time $t_{\text {awake }}$ should be large enough so that any sensor in the outmost band would be able to receive any message sent by the sink and destined to this band. This would help each sensor in the outmost band to check whether it has been designated by the sink to participate in $k$-covering its band.

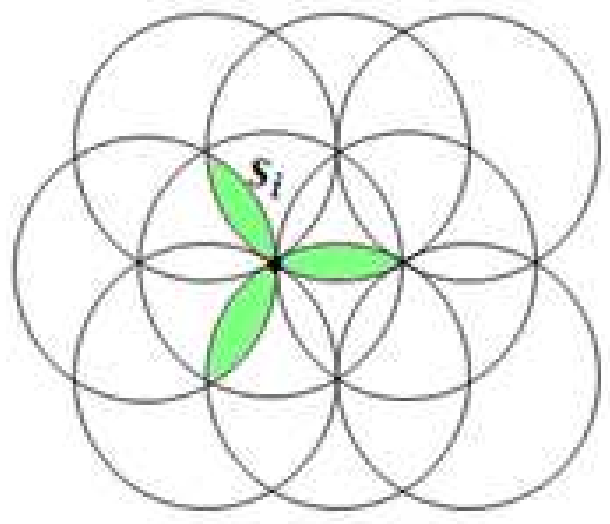

Figure 4: Three-lens flower of $s_{i}$

\subsubsection{Distributed $\boldsymbol{k}$-Coverage Protocol}

In our distributed protocol (PR-Het-DCC ${ }_{k}$ ), each sensor $s_{i}$ randomly slices its sensing range into six overlapping Reuleaux triangles of width equal to $r_{i}$, the radius of $s_{i}$ 's sensing range. Based on the result of Theorem 1, a sensor $s_{i}$ randomly picks a three-lens flower (as shown in Figure 4) and checks whether its sensing range is $k$-covered. A sensor starts first by choosing the sensors located in the three lenses of the selected three-lens flower to remain active and $k$-cover its sensing range based on their remaining energy and the radii of their sensing ranges. Specifically, a sensor $s_{i}$ selects $k$ sensors from each of the lenses of the three-lens flower whose sensing ranges' radii are at least equal to $r_{i}$, the radius of the sensing range of $s_{i}$. Then, it activates them by sending AWAKE messages. When a sensor receives an AWAKE message, it becomes active and broadcasts a NOTIFICATION message to inform all its neighbors. Each sensor keeps track of its active neighbors. Also, a sensor will turn itself active if its sensing range is not $k$-covered.

First, recall that all the sensors located in the same band have the same capabilities, including their sensing range. Our distributed connected $k$-coverage protocol, denoted by PRHet-DCC $k$, requires that a sensor $s_{j}$ located in a band $b_{j}$ randomly decompose its sensing range into six overlapping Reuleaux triangles of width $r_{j}$ as computed in equation (1) (i.e., $r_{j}=r_{\max }-(j-1) \times d_{w}$ ), where $r_{j}$ is the radius of $s_{j}$ 's sensing range (or the width of the band $b_{j}$ ). Furthermore, a sensor $s_{j}$ would select from its three-lens flower (as shown in Figure 3) only the sensors that are located either in its band $b_{j}$ or in its adjacent band $b_{j-1}$ to $k$-cover its sensing range. Indeed, the sensors located in $b_{j-1}$ have higher sensing range than $s_{j}$ and thus will be able to participate in the $k$-coverage of $s_{j}$ 's sensing range when they are selected. This means that a sensor $s_{j}$ would select sensors from its band $b_{j}$ which have the same power as $s_{j}$ (especially, their sensing range) or more powerful than $s_{j}$ (i.e., from the band $b_{j-1}$ ) to ensure $k$-coverage of its sensing range. This helps cope with the problem of under $k$-coverage, where some areas of the field are less than $k$-covered. This problem may arise if $s_{j}$ chooses sensors whose radius of sensing range less than $r_{j}$. 
Next, we propose a few data collection schemes. In the first set, we assume all the sensors are immobile, while in the second set, we consider mobility and investigate its impact.

\section{Immobile Data Collection}

In this section, we propose three data collection protocols on top of connected $k$-coverage configurations in WSNs. While the first two protocols use a deterministic approach, the third one is based on an opportunistic approach, which copes with the shortcomings of the former method. Specifically, our opportunistic forwarding approach will combine the use of both deterministic forwarding schemes along with other parameters to be specified shortly. Furthermore, we assume that all the sensors and the sink do not move (i.e., immobile).

\subsection{Deterministic Forwarding Schemes}

We assume that the sink is located at the center of the circular field. Indeed, Luo and Hubaux [17] proved that the center is the optimum position for a sink in terms of energyefficient data collection. Moreover, each sensor node is aware of the location information of each of its neighboring sensor nodes, as well as the location of the sink. This type of information can be gathered by each sensor node at the beginning of deployment of the sensor nodes. All the sensors located at the different bands are supposed to forward their sensed data to the single static sink using multi-hop communication path through other intermediate sensors. In this type of deterministic geographic forwarding approach [14], a sensor node chooses its next forwarder deterministically and based on some metrics, namely distance to the source sensor and remaining energy. More precisely, we consider two deterministic schemes, which we discuss below in details.

\subsubsection{Scheme 1: Short-Range Data Forwarding}

First, each sensor node $s_{i}$ prefers to select one of its neighboring sensors that is active and has the highest remaining energy (HRE) as its next forwarder. Obviously, this forwarder is located in the area between $s_{i}$ and the sink so the sensed data is forwarded progressively toward the sink (i.e., phenomenon known as positive progress). This design decision ensures that all the neighboring sensors of a sensor node $s_{i}$ are equally likely to participate in the forwarding process. In addition, it leads to load balancing in the entire network. Second, sensor node $s_{i}$ chooses one of its active closest neighboring sensors (CNS) to act as next forwarder. This design decision is motivated by the fact that the energy consumption of a sensor node due to data transmission is proportional to the distance a message would travel from a sender to a receiver. Hence, sensor node $s_{i}$ aims at minimizing its energy consumption, thus, extending its lifetime. Third, sensor node $s_{i}$ prefers a neighboring sensor that lies as close as possible to the shortest path (SP) between $s_{i}$ and the sink. This latter choice ensures that all selected forwarders will not deviate much from the shortest path between sensor node $s_{i}$ and the sink, thus, reducing the delay incurred by a message to reach the sink. To account for these three attribute, namely highest energy reserve, closeness, and shortest path, we propose a metric, which is denoted by HRE_CNS_SP $\left(s_{j}\right)$ and defined as:

$$
\begin{gathered}
H R E \_C N S \_S P\left(s_{j}\right)=\left[E_{r e m}\left(s_{j}\right) / \delta\left(s_{i}, s_{j}\right)\right] \times \\
{\left[\delta\left(s_{i}, \operatorname{sink}\right) /\left(\delta\left(s_{i}, s_{j}\right)+\delta\left(s_{j}, \sin k\right)\right)\right]}
\end{gathered}
$$

where $s_{j}$ is a neighboring sensor of $s_{i}$ (i.e., $s_{j} \in N S\left(s_{i}\right)$ ), $E_{r e m}\left(s_{k}\right)$ stands for the remaining energy of sensor node $s_{k}$, and $\delta\left(s_{u}, s_{v}\right)$ stands for the Euclidean distance between sensor nodes $s_{u}$ and $s_{v}$. It is easy to check that

$$
\delta\left(s_{i}, \sin k\right) /\left(\delta\left(s_{i}, s_{j}\right)+\delta\left(s_{j}, \sin k\right)\right) \leq 1
$$

It is clear that the maximum value 1 is reached when $s_{j}$ lies on the shortest path (or line segment) between $s_{i}$ and the sink. In summary, sensor node $s_{i}$ selects its neighboring sensor $s_{n f}$ as its next forwarder such that $H R E \_C N S \_S P\left(s_{n f}\right)$ is the highest value among all the neighboring sensors $s_{j}$ of sensor node $s_{i}$. In other words,

$$
\begin{gathered}
H R E \_C N S \_S P\left(s_{n f}\right)=\max \left\{\left[E_{\text {rem }}\left(s_{j}\right) / \delta\left(s_{i}, s_{j}\right)\right] \times\left[\delta\left(s_{i}, \operatorname{sink}\right) /\right.\right. \\
\left.\left.\left(\delta\left(s_{i}, s_{j}\right)+\delta\left(s_{j}, \operatorname{sink}\right)\right)\right] \mid s_{j} \in N S\left(s_{i}\right)\right\}
\end{gathered}
$$

Notice that HRE_CNS_SP increases inversely proportionally to $\delta\left(s_{i}, s_{j}\right)$. That is, the closest sensor $s_{j}$ to $s_{i}$ will lead to maximize HRE_CNS_SP as the transmission distance of sensor $s_{i}$ gets smaller. This feature is highly desirable for low-powered sensors. Indeed, this is the fundamental driving force behind short-range data forwarding. Any selected next forwarder will apply the same algorithm to forward sensed data to the sink.

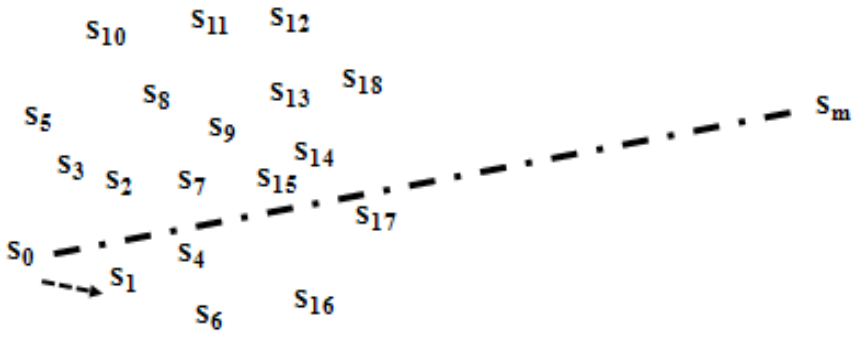

(a)

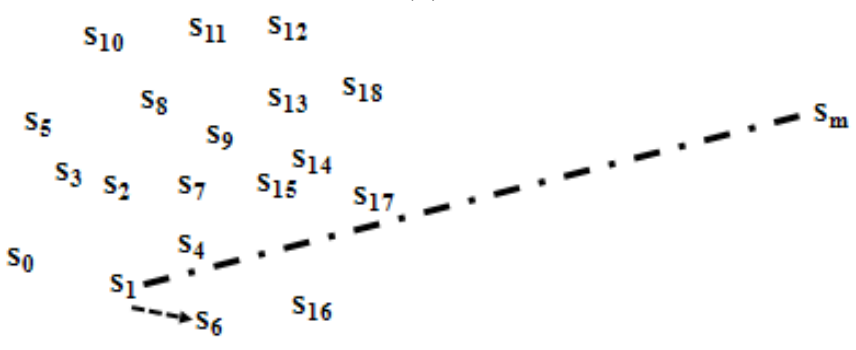

(b)

Figure 5: Illustration of Scheme 1

Figure 5 illustrates the main idea behind Scheme 1. Let $s_{m}$ denote the sink and assume the source sensor $s_{0}$ wants to send its data to the sink. Assume that the neighboring sensor set of $s_{0}$ includes $s_{1}, s_{2}, s_{3}, \ldots, s_{18}$. As it can be seen, the 
closest sensor to $s_{0}$ is $s_{1}$. Also, $s_{1}$ is the closest sensor to the line connecting $\mathrm{s}_{0}$ to $s_{m}$. Moreover, assume that $s_{1}$ has the highest remaining energy compared to all other neighboring sensors of $s_{0}$. Clearly, using Scheme $1, s_{0}$ would pick $s_{1}$ as its nest forwarder as shown in Figure 5a. The latter repeats the same process to forward data toward the sink $s_{m}$. Now, $s_{2}, s_{4}$, $s_{6}$, and $s_{7}$ are the closest neighboring sensors to $s_{1}$. Assume that $s_{6}$ has the highest value HRE_CNS_SP $\left(s_{6}\right)$ compared to those associated with $s_{2}, s_{4}$, and $s_{7}$. In this case, $s_{1}$ would pick $s_{6}$ as its nest forwarder as shown in Figure $5 \mathrm{~b}$

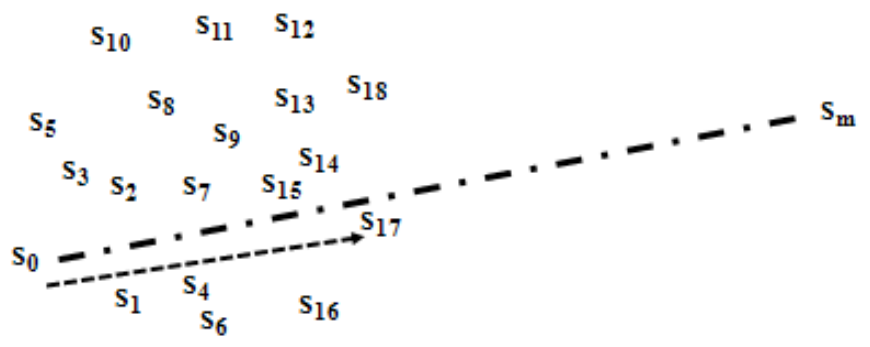

Figure 6: Illustration of Scheme 2

\subsubsection{Scheme 2: Long-Range Data Forwarding}

This forwarding scheme is similar to the above-mentioned one except that sensor node $s_{i}$ prefers one of its farthest neighboring sensors (FNS) to act as next forwarder. To account for these three attributes, namely highest energy reserve, fartherness, and shortest path, we introduce a metric, which is denoted by $H R E \_F N S \_S P\left(s_{j}\right)$ and defined as:

$$
\begin{gathered}
H R E \_F N S \_S P\left(s_{j}\right)=\left[E_{r e m}\left(s_{j}\right) / \delta\left(s_{j}, \sin k\right)\right] \times\left[\delta\left(s_{i}, \operatorname{sink}\right) /\left(\delta \left(s_{i},\right.\right.\right. \\
\left.\left.\left.s_{j}\right)+\delta\left(s_{j}, \sin k\right)\right)\right]
\end{gathered}
$$

Thus, sensor node $s_{i}$ selects its neighboring sensor $s_{n f}$ as its next forwarder such that $H R E_{-} F N S \_S P\left(s_{n f}\right)$ is the highest value among all the neighboring sensors $s_{j}$ of sensor node $s_{i}$. In other words,

$$
\begin{gathered}
H R E \_F N S \_S P\left(s_{n f}\right)=\max \left\{\left[E_{\text {rem }}\left(s_{j}\right) / \delta\left(s_{j}, \operatorname{sink}\right)\right] \times\left[\delta\left(s_{i}, \operatorname{sink}\right) /\right.\right. \\
\left.\left.\left(\delta\left(s_{i}, s_{j}\right)+\delta\left(s_{j}, \sin k\right)\right)\right] \mid s_{j} \in N S\left(s_{i}\right)\right\}
\end{gathered}
$$

Now, one can see that HRE_FNS_SP increases inversely proportionally to $\delta\left(s_{j}, \sin k\right)$. That is, the closest sensor $s_{j}$ to the sink (i.e., the farthest sensor from $s_{i}$ ) will help maximize $H R E \_F N S \_S P$ as the transmission distance of sensor $s_{i}$ gets larger, thus, minimizing the number of hops (or intermediate relay nodes) between the source sensors and the sink. This is a highly desirable feature for time-critical sensing applications that have hard deadlines. Indeed, this is the fundamental driving force of long-range data forwarding. While Scheme 1 is a short-range data forwarding protocol, Scheme 2 is a long-range data forwarding protocol. Compared to Scheme 1, one can easily notice that Scheme 2 yields higher energy consumption by each sensor. However, it progresses the sensed data faster toward the sink, thus, incurring lower delay. Clearly, Scheme 2 is more suitable for sensing applications where delay is a very critical attribute to the effectiveness of the underlying WSN.
Figure 6 provides an illustrative example of Scheme 2. Suppose that the source sensor $s_{0}$ wants to transmit its sensed data to the sink $s_{m}$, and let $\left\{s_{1}, s_{2}, s_{3}, \ldots, s_{18}\right\}$ be the neighboring sensor set of $s_{0}$. The farthest neighboring sensors to $s_{0}$ are $s_{12}, s_{16}, s_{17}$, and $s_{18}$. Notice that $s_{17}$ lies closer to the line connecting $\mathrm{s}_{0}$ to $s_{m}$. Furthermore, assume that $s_{17}$ has the highest remaining energy compared to all other neighboring sensors of $s_{0}$, and that $s_{17}$ has the highest value $H R E \_F N S \_S P\left(s_{17}\right)$ compared to those associated with $s_{12}, s_{16}$, and $s_{18}$. Based on Scheme 2, $s_{0}$ would pick $s_{17}$ as its nest forwarder as shown in Figure 6. Obviously, $s_{17}$ would apply the same technique when selecting its next forwarder.

\subsection{Opportunistic Forwarding Scheme}

Here, we show that both of the deterministic approaches, namely Schemes 1 and 2, suffer from two major problems due to sensor heterogeneity and duty-cycling. Then, we describe our third data forwarding scheme, which is opportunistic, in order to cope with these two problems.

\subsubsection{Major Problems with Deterministic Schemes}

As it can be noticed, the first problem is that the two above schemes do not benefit from the heterogeneity of the sensors in their respective bands. Indeed, regardless of the bands they belong to, all sensors use the same forwarding scheme: Either short-range data forwarding (Scheme 1) or long-range data forwarding (Scheme 2). It is important to design an adaptive data collection scheme in which data forwarding depends on our layered architecture, where sensors in different bands have different features. To account for sensor heterogeneity, we propose a hybrid forwarding scheme which benefits from Scheme 1 and Scheme 2. Precisely, we require that the sensors in the outer bands forward sensed data over short distances, which helps them save their energy. In fact, those sensors have lower energy and, thus, should have lower forwarding load using short-range data forwarding. Also, the sensors in the inner bands send data over long distances. Indeed, those sensors have higher energy, and, thus, should have higher forwarding load using long-range data forwarding. This helps bypass the sensors nearer the sink, thus, saving their energy.

The second problem is that both of Scheme 1 and Scheme 2 use deterministic forwarding where a sensor chooses a next best forwarder based on those three metrics and forwards data to it. As can be seen, the next forwarder is determined $a$ priori. Notice that the sensors that are selected to $k$-cover the circular field are the only ones that will be responsible for forwarding data to the sink on behalf of others. Moreover, those selected sensors will be active for one round. Some of them (if not all of them) may not be selected in the next round to participate in the $k$-coverage the circular field, and, thus, remain off (or inactive). Thus, a sensor that has been selected to act as next forwarder for a current round may not remain active in the next one. Thus, because of duty-cycling, sensors holding data to be forwarded to the sink and using deterministic forwarding are not totally certain that their 
currently awake sensing neighbors would remain awake after data is being forwarded. Clearly, duty-cycling introduces uncertainty at the sender side when selecting a next best forwarder. To cope with this problem, we require the sensors to use opportunistic forwarding [6] where a next best forwarder is decided on-the-fly and after the data is sent. However, with opportunistic forwarding, several active sensors may hear the transmitted data, thus creating high contention at the receiver side to select a next best forwarder. Thus, it is important to find a trade-off between uncertainty due to duty-cycling with deterministic forwarding, and contention due to opportunistic forwarding. Next, we describe our hybrid forwarding approach in details.

\section{Algorithm : Scheme 3 \\ Procedure Forward_Data \\ Begin \\ 1. $s_{f}$ forwards sensed data to the sink $s_{m}$ using Scheme 1 if $s_{f}$ 's band's id is greater than or equal to $\lceil n / 2\rceil$; otherwise, $s_{f}$ uses Scheme 2 ( $n$ being the number of bands in the circular deployment field) \\ 2. $s_{f}$ sends an ACK to the previous forwarder and $m-1$ candidate forwarders \\ End \\ Begin \\ /* This code section is run by a sender or current forwarder $s_{i} * /$}

1. Sort all potential forwarders in a descending order of their remaining energy and break their tie using their closeness to the sink $s_{m}$

2. Select the first three candidate forwarders, store them in a sensed data packet, and broadcast it using Scheme 1 if $s_{i}$ 's band's id is greater than or equal to $\lceil n / 2\rceil$; otherwise, $s_{i}$ uses Scheme 2

/* This code section is run by a candidate next forwarder $s_{c f} * 1$

3. Exchange messages between the $m$ candidate next forwarders

4. If $s_{c f}$ has the highest remaining energy Then

5. $s_{c f}$ calls Forward_Data

End

Figure 7: Scheme 3 (Joint $k$-coverage and adaptive hybrid forwarding protocol)

\subsubsection{Scheme 3: Adaptive Hybrid Forwarding}

Our proposed forwarding scheme, as described in Figure 7, is adaptive in the sense it exploits the abovementioned design decision, where sensors located away from the sink in the outer bands use short-range data forwarding (or Scheme 1), while sensors belonging to the inner bands use long-range data forwarding (or Scheme 2). Without loss of generality, all sensors in bands $1,2, \ldots,\lceil n / 2\rceil-1$ use Scheme 2, while all sensors in bands $\lceil n / 2\rceil,\lceil n / 2\rceil+1, \ldots, n$ use Scheme 1 , where $n$ is the number of bands forming the circular field and which can be computed using equations (2) and (3) above.
Also, it is hybrid as it takes advantages of deterministic and opportunistic forwarding approaches in order to achieve good data forwarding performance in terms of data delivery ratio, delay, and control overhead. Precisely, a sensor node $s_{i}$ specifies in its sensed data packet the $i d$ 's of $m$ active candidate next forwarders $\left(s_{c f}\right)$ from its communication neighboring set, where $m>1$. Upon receiving this data packet, the $m$ designated sensors exchange short messages including the current value of their remaining energy, provided they are active. The one with the highest value is officially selected as the next forwarder $\left(s_{f}\right)$ of the received data packet. Intuitively, one of the $m$ candidate next forwarders that is active and has the largest remaining energy is selected as the next forwarder. After forwarding this data packet, $s_{f}$ sends an acknowledgment message to the sensor node $s_{i}$ and the $m-1$ other candidate next forwarders letting them know that the data packet has been forwarded. It is worth noting that these $m$ candidate next forwarders are located in one of the lenses of the sensing disks of sensor node $s_{i}$. Thus, they are able to communicate with each other. To minimize the energy consumption due to the communication between these $\kappa$ candidate next forwarders, we simply set $m=3$, which seems to be a reasonable value. Thus, our adaptive hybrid forwarding scheme (Scheme 3), which is shown in Figure 8, requires little communication between those $m$ candidate next forwarders.

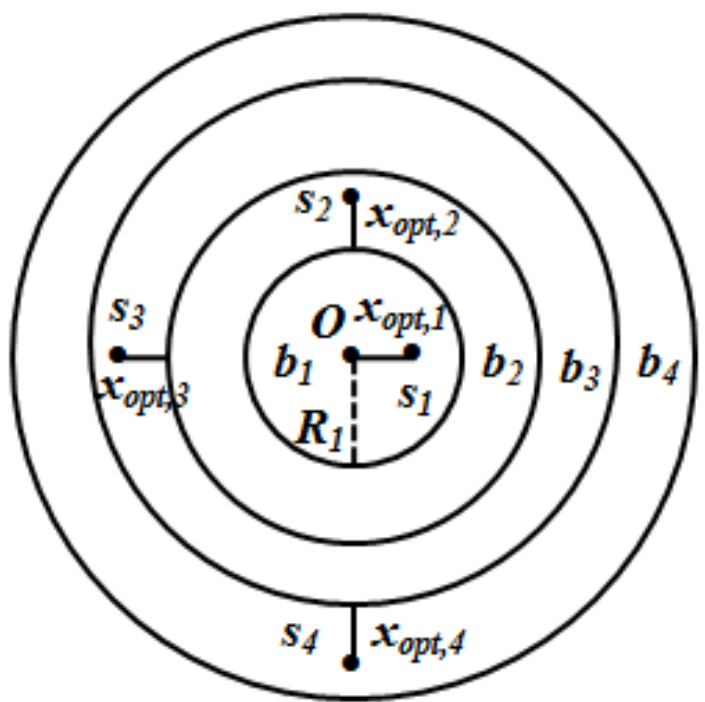

Figure 8: Width of mobility band

\section{Mobile Data Collection}

In this section, we study sink mobility during data collection in order to save the energy of the sensors, thus, extending the whole network lifetime. Based on this study, we propose a data collection protocol using a single mobile sink.

\subsection{Optimal Sink Mobility}

Here, we investigate the mobility of the sink within each band in order to collect data from the sensors. Our goal is to 
minimize the total energy consumption due to data transmission, data reception, and sink mobility.

As it can be seen, the shortest mobility trajectory of the sink within a band $b_{j}$ of width $w_{j}$ is a circle of radius $\rho=$ $\sum_{i=1 . . j-1} w_{i}+x$ as shown in Figure 8. This circular trajectory splits band $b_{j}$ into two sub-bands, namely $b_{j 1}$ and $b_{j 2}$. Thus, the mobile sink $s_{0}$ collects data from the sensors located in bands $b_{j 1}$ and $b_{j 2}$ of widths $x$ and $w_{j}-x$, respectively. To minimize the energy consumption due to data transmission, each sensor $s_{i}$ submits its data directly to the sink $s_{0}$ only when the line segment connecting the respective locations of $s_{i}$ and $s_{0}$, say $\xi_{i}$ and $\xi_{j}$, is orthogonal to the tangential passing by $\xi_{j}$ located at the perimeter of the mobility circle of $s_{0}$. First, let us compute the average transmission distance between sensor $s_{i}$ in band $b_{j 1}$ and the mobile sink $s_{0}$. The following lemma [3] computes this distance. Also, for the sake of completeness, we provide the proof of this lemma.

Lemma 4 [3]: The average transmission distance, denoted by $d_{a v g, j 1}$, between a sensor $s_{i}$ in band $b_{j 1}$ whose width is $x$, and the mobile sink $s_{0}$ is given by

$$
d_{\text {avg, } 11}=\frac{1}{3} x
$$

Proof: Consider band $b_{j 1}$ whose width is equal to $x$, where each sensor is located at position $(0, x)$ whereas the mobile sink $s_{0}$ is positioned at location $x_{l}=(0, x)$. All the locations of the sensors form a uniform distribution. We pick a sensor $s_{i}$ randomly located at $x_{i}=(0, x)$. The average Euclidean distance (i.e., average transmission distance) between $x_{i}$ and $x_{l}$ is given by

$$
\begin{gathered}
d_{\text {avg }, j 1}=\frac{\int_{[0, x]} \int_{[0, x]}\left|x_{i}-x_{l}\right| d x_{i} d x_{l}}{\int_{[0, x]} \int_{[0, x]} d x_{i} d x_{l}} \\
\int_{[0, x]} \int_{[0, x]} d x_{i} d x_{l}=\int_{0}^{x} \int_{0}^{x_{l}} d x_{i} d x_{l}=\frac{1}{2} x^{2}
\end{gathered}
$$

Given that $x_{l}=(0, x)$ and $x_{i}=(0, x)$, we deduce that $\left|x_{i}-x_{l}\right|=x_{l}-x_{i}$. Thus, we have

$$
\begin{gathered}
\int_{[0, x]} \int_{[0, x]}\left|x_{i}-x_{l}\right| d x_{i} d x_{l} \\
=\int_{0}^{x} \int_{0}^{x_{l}}\left(x_{l}-x_{i}\right) d x_{i} d x_{l}=\frac{1}{6} x^{3}
\end{gathered}
$$

We conclude that $d_{\text {avg }, j 1}\left(x_{i}, x_{l}\right)=\frac{1}{3} x$.

Notice that sensors in $b_{j 1}$ transmit their data to $m p s_{1}$ over distances ranging between 0 and $x_{o p t}$, those in $b_{i, 2}$ send their data to $m p s_{1}$ over distances ranging between 0 and $R-x_{o p t}$. Based on the above lemma, it is clear that the average transmission distance between a sensor $s_{i}$ in band $b_{j 2}$ and the mobile sink $s_{0}$, denoted by $d_{a v g, j 2}$, can be computed as follows:

$$
d_{\text {avg }, j 2}=\frac{1}{3}(R-x)
$$

Next, we compute the optimum value of $x$, denoted by $x_{\text {opt }}$, which helps minimize the average total energy consumption due to data transmission and reception as well as sink mobility. Theorem 2 computes the value of $x_{o p t}$, which we refer to as the width of mobility band.

Theorem 2: The width of mobility band that corresponds to the optimum mobility trajectory of the mobile sink $s_{0}$ inside band $b_{j}$ is given by

$$
x_{o p t, j}=\frac{-B+\sqrt{\Delta}}{2 A}
$$

with $u, v$, and $\Delta$ being defined as follows:

$$
\begin{gathered}
A=\frac{2 \lambda \pi \kappa \varepsilon R_{j}}{3} \\
B=\frac{2 \lambda \pi \kappa \varepsilon}{9}\left(\left(\sum_{t=1 . . j} w_{t}\right)^{2}+\left(\sum_{t=1 . j-1} w_{t}\right)^{2}\right. \\
\left.+4 \epsilon R_{j} \sum_{t=1 . . j-1} w_{t}-R_{j}^{2}\right) \\
C=2 \pi e_{\text {move }} \\
\Delta=B^{2}-4 A C
\end{gathered}
$$

where $w_{j}$ is the width of band $b_{j}$ and $R_{j}$ is the radius of the communication range of the sensors located in band $b_{j}$.

Proof: Let us compute the average total energy consumption for data transmission, data reception, and sink mobility. Assume that $e_{\text {move }}$ is the average energy spent when the mobile sink moves a unit of distance. Thus, the energy spent during sink mobility is computed as

$$
E_{\text {avg-mob-bj}}(x)=2 \pi e_{\text {move }}\left(x+\sum_{t=1 . . j-1} w_{t}\right)
$$

where $w_{t}$ is the width of band $b_{t}$, for $t=1 . . j-1$. Given that the sensors are uniformly distributed in each band, the number of sensors $n_{j 1}$ and $n_{j 2}$ in sub-bands $b_{j 1}$ and $b_{j 2}$, respectively, are given by

$$
\begin{gathered}
n_{j 1}=\lambda \pi\left(\left(x+\sum_{t=1 . . j-1} w_{t}\right)^{2}-\left(\sum_{t=1 . . j-1} w_{t}\right)^{2}\right) \\
n_{j 2}=\lambda \pi\left(\left(\sum_{t=1 . . j} w_{t}\right)^{2}-\left(x+\sum_{t=1 . . j-1} w_{t}\right)^{2}\right)
\end{gathered}
$$

where $\sigma_{1}=\sum_{t=1 . . j-1} w_{t}, \sigma_{2}=x+\sum_{t=1 . . j-1} w_{t}$, and $\sigma_{3}=$ $\sum_{t=1 . . j} w_{t}$ are the inner radius of sub-band $b_{j l}$, outer radius of sub-band $b_{j 1}$ (also, inner radius of sub-band $b_{j 2}$ ), and outer radius of sub-band $b_{j 2}$, respectively. Using Lemma 4 and Corollary 1 stated above, the total energy spent in data transmission and reception is given by: 
$E_{a v g-t r x-b j}(x)=n_{j 1} E_{a v g-t r x-b j 1}(x)+n_{j 2} E_{a v g-t r x-b j 2}(x)$ where:

$$
\begin{aligned}
& E_{a v g-t r x-b j 1}(x)=\kappa\left(\varepsilon d_{a v g, j 1}{ }^{2}+2 E_{e l e c}\right) \\
& =\kappa\left(\varepsilon\left(\frac{x}{3}\right)^{2}+2 E_{-} \text {elec }\right) \\
& E_{a v g-t r x-b j 2}(x)=\kappa\left(\varepsilon d_{a v g, j 2}^{2}+2 E_{e l e c}\right) \\
& =\kappa\left(\varepsilon\left(\frac{R-x}{3}\right)^{2}+2 E_{-} \text {elec }\right)
\end{aligned}
$$

Therefore, we have:

$$
E_{a v g-t o t-b j}(x)=E_{a v g-m o b-b j}(x)+E_{a v g-t r x-b j}(x)
$$

To compute the optimum value of $x, x_{\text {opt }}$, in order to minimize the average total energy consumption, we solve the following derivative:

$$
\frac{\partial E_{a v g-t o t-b j}(x)}{\partial x}=0
$$

With a little algebra, we obtain the following unique closedform solution:

$$
x_{o p t, j}=\frac{-B+\sqrt{\Delta}}{2 A}
$$

with $A, B$, and $\Delta$ being defined as follows:

$$
\begin{gathered}
A=\frac{2 \lambda \pi \kappa \varepsilon R_{j}}{3} \\
B=\frac{2 \lambda \pi \kappa \varepsilon}{9}\left(\left(\sum_{t=1 . . j} w_{t}\right)^{2}+\left(\sum_{t=1 . . j-1} w_{t}\right)^{2}\right. \\
\left.+4 \epsilon R_{j} \sum_{t=1 . . j-1} w_{t}-R_{j}{ }^{2}\right) \\
C=2 \pi e_{\text {move }} \\
\Delta=B^{2}-4 A C
\end{gathered}
$$

\subsection{Scheme 4: Sink Mobility-Based Data Collection}

Recall that there is only one mobile sink, denoted by $m s_{1}$, for data gathering. As mentioned earlier, the mobile sink $m s_{1}$ adopts a circular trajectory in its mobility in each band for data collection from all the sensors inside the underlying band. More precisely, when gathering data from band $b_{j}$, the mobile sink $m s_{1}$ moves inside $b_{j}$ on a circle of radius $\Psi_{j}$, which is computed as follows:

$$
\Psi_{j}=\sum_{t=1 . . j-1} R_{t}+x_{o p t, j}
$$

with $R_{t}$ being the radius of the communication range of the sensors located inside band $b_{t}$. Then, $m s_{1}$ follows a linear trajectory to move to the next band $b_{j+1}$, where it will have a similar circular trajectory on a circle of radius $\Psi_{j+1}$. This alternation of circular movement and linear movement of the mobile sink repeats until it visit the last band $b_{n}$. Then, it follows a linear movement toward the center of the field of interest until it reaches the circle of radius $x_{o p t, 1}$ (as defined in Theorem 2), which is located in the first band $b_{1}$. The mobile sink $m s_{1}$ uses the same trajectories in the next round as in the previous one to collect data from each of the visited bands, $b_{1}, b_{2}, \ldots, b_{n}$. Figure 9 illustrates this mixed movement of circular trajectory and linear trajectory of the mobile sink during any round of data gathering with respect to two consecutive bands, namely $b_{j}$ and $b_{j+1}$. We assume that during any round of data gathering, the single mobile sink $m s_{1}$ has a constant mobility speed between $v$, and stops at some locations on its circular trajectories to collect data.

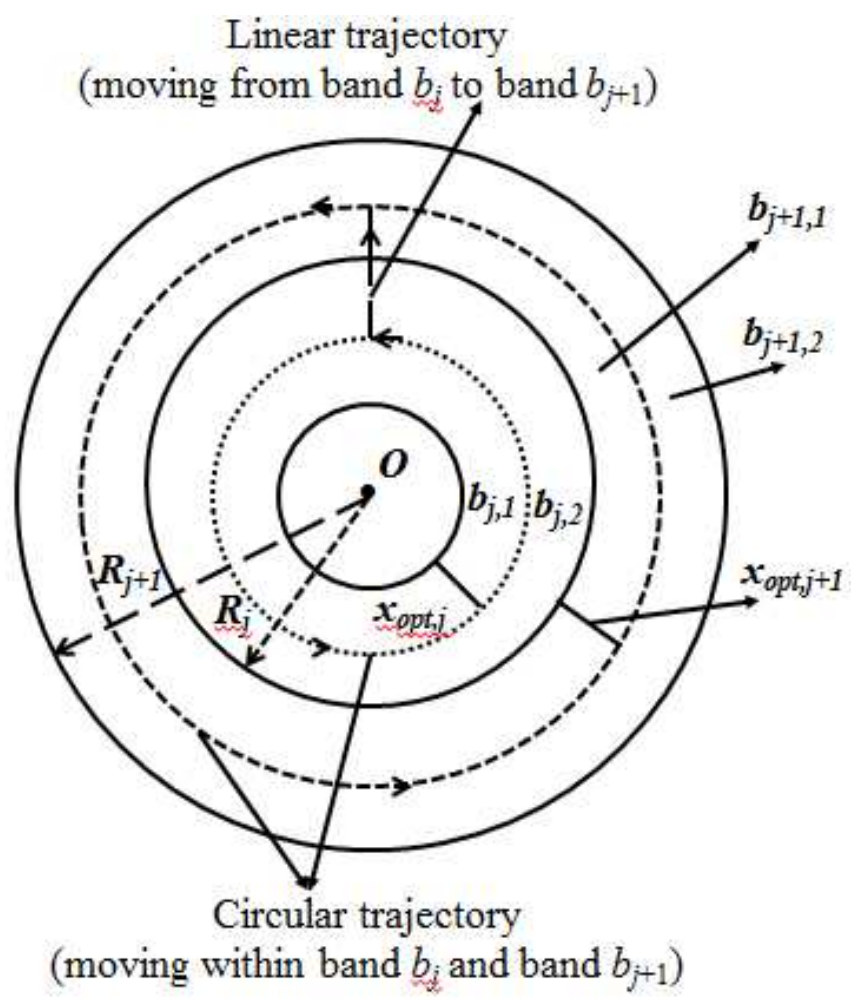

Figure 9: Linear and circular trajectories of sink mobility

\section{Performance Evaluation}

In this section, we present a variety of simulation results of our $k$-coverage and data collection protocols for various network setups using a high-level simulator written in $C$. First, we specify our simulation environment. Then, we discuss our simulation-based findings with respect to several metrics and scenarios, such as the number of active sensors as a function of the degree of coverage $k$; homogeneous $k$ coverage compared to heterogeneous $k$-coverage; random sensor deployment against pseudo-random sensor deployment; centralized $k$-coverage compared to distributed $k$-coverage; delay as a function of the coverage degree $k$; data delivery ratio depending on the coverage degree $k$; and adaptive hybrid forwarding using a static sink compared to mobile sink-based data collection.

\subsection{Simulation Setup}


We consider a circular field of radius $D=1000 \mathrm{~m}$. We use the energy model in [29], where the sensor energy consumption in transmission, reception, idle, and sleep modes are $60 \mathrm{~mW}, 12 \mathrm{~mW}, 12 \mathrm{~mW}$, and $0.03 \mathrm{~mW}$, respectively. Following [31], the energy required for a sensor to stay idle for 1 second is equivalent to one unit of energy. Given that the sensors are heterogeneous, we assume that the initial energy of each sensor is between 60 Joules and 80 Joules. Also, we assume that the mobility speed of the sink is $v=1 \mathrm{~m} / \mathrm{s}$ during any round of data gathering. All simulations are repeated 200 times and the results are averaged.

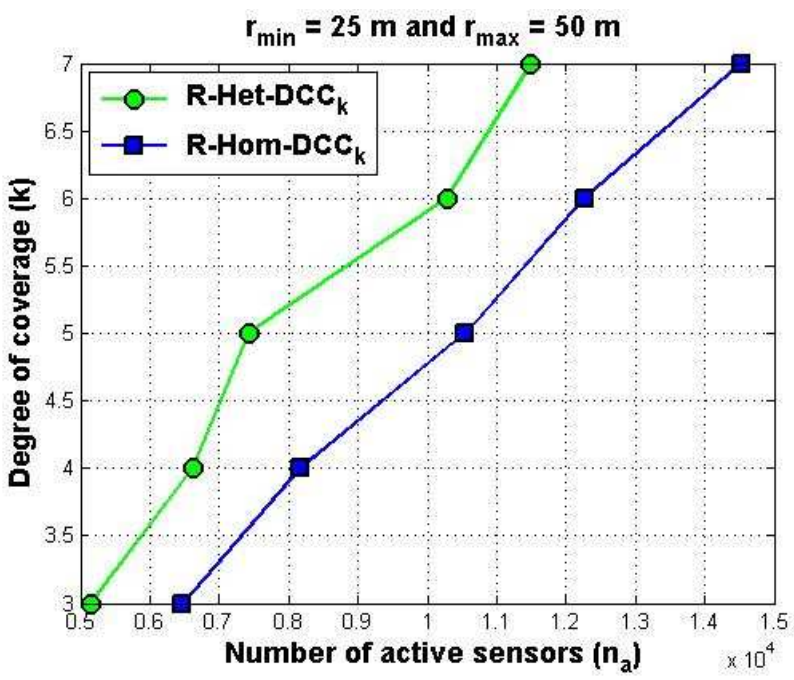

Figure 10: R-Het-DCC ${ }_{k}$ vs. R-Hom-DCC ${ }_{\mathrm{k}}$ (Number of active sensors)

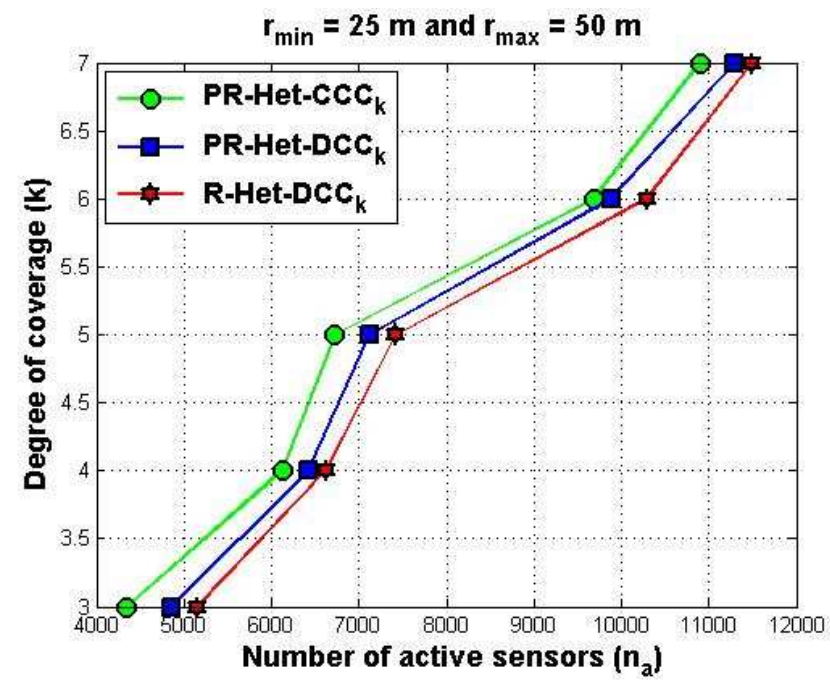

Figure 11: Comparing PR-Het-CCC ${ }_{k}$, PR-Het-DCC ${ }_{k}$, and

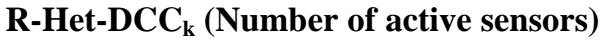

\subsection{Simulation Results}

In Figure 10, we compare both of our distributed connected $k$-coverage protocols, namely $\mathrm{R}$-Hom-DCC $\mathrm{D}_{k}$, where the sensors are homogeneous and randomly deployed, and R-
Het-DCC ${ }_{k}$, where the sensors are heterogeneous and randomly deployed ("R" stands for "random"). For R-Hom$\mathrm{DCC}_{k}$, we assume that the sensing range of all the sensors is $r=37.5 \mathrm{~m}$ and that the initial energy of each sensor is 70 Joules. On the other hand, for R-Het-DCC ${ }_{k}$, the sensing range of the sensors is between $r_{\text {min }}=25 \mathrm{~m}$ and $r_{\max }=50 \mathrm{~m}$ with mean value equal to $37.5 \mathrm{~m}$, and the initial energy of each sensor is between 60 Joules and 80 Joules with mean value equal to 70 Joules. As can be seen from Figure 10, RHet-DCC $C_{k}$ outperforms R-Hom-DCC ${ }_{k}$ as it requires less number of sensors for any coverage degree $k$. Obviously, the presence of more powerful sensors helps ensure $k$-coverage of a field of interest with less number of active sensors.

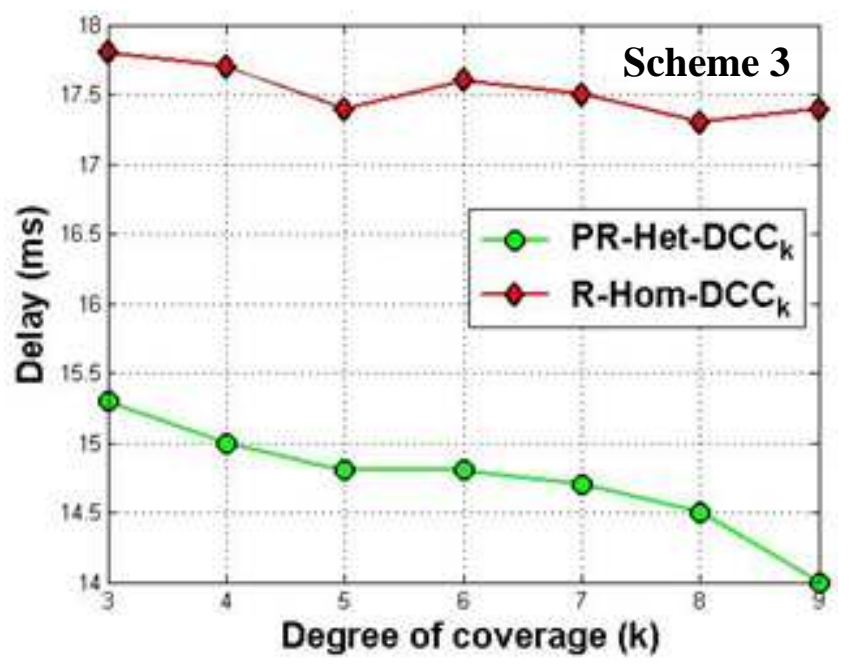

Figure 12: Comparing PR-Het-DCC ${ }_{k}$ and R-Hom-DCC $\mathrm{k}_{\mathrm{k}}$ (Delay)

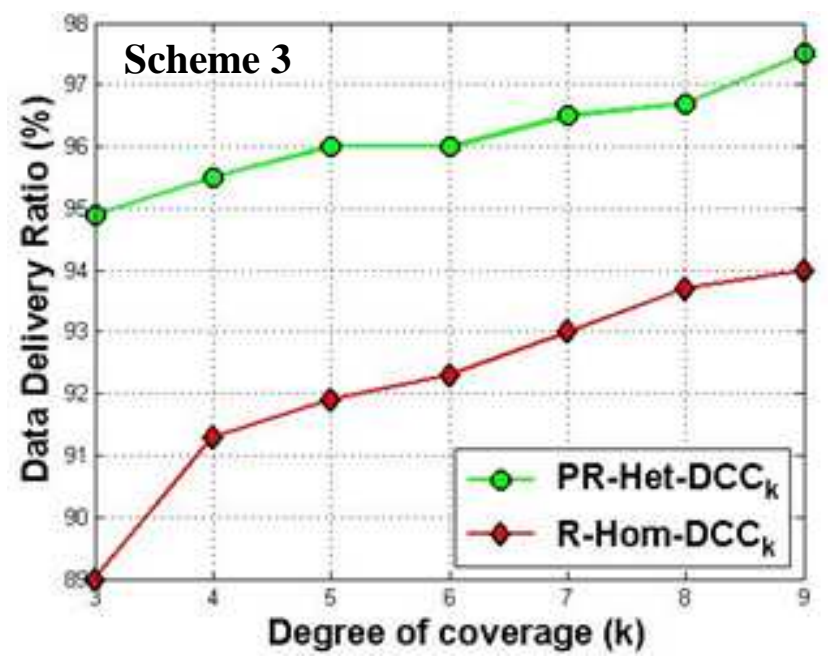

Figure 13: Comparing PR-Het-DCC ${ }_{k}$ and R-Hom-DCC ${ }_{\mathrm{k}}$ (Data delivery ratio)

Figure 11 shows that our centralized protocol PR-Het$\mathrm{CCC}_{k}$ outperforms our distributed protocol PR-Het-DCC $\mathrm{C}_{k}$ (both for pseudo-random deployment of heterogeneous sensors). This is mainly due to the absence of coordination in 
PR-Het-CCC $C_{k}$ between adjacent sensors to achieve $k$ coverage of their sensing ranges. The sink only needs to keep track of the locations of all the sensors as well as their remaining energy. Although there is some energy consumed to forward all requests of the sink to their destination sensors, it is less than the energy needed for the coordination between the sensors to select a small number of sensors to $k$-cover the field. Also, we found that PR-Het-DCC $C_{k}$ has better results than our distributed protocol R-Het-DCC $\mathrm{C}_{k}$ for purely random deployment of heterogeneous sensors. This shows the impact of slicing the field into bands and deploying sensors in those bands based on their capabilities.

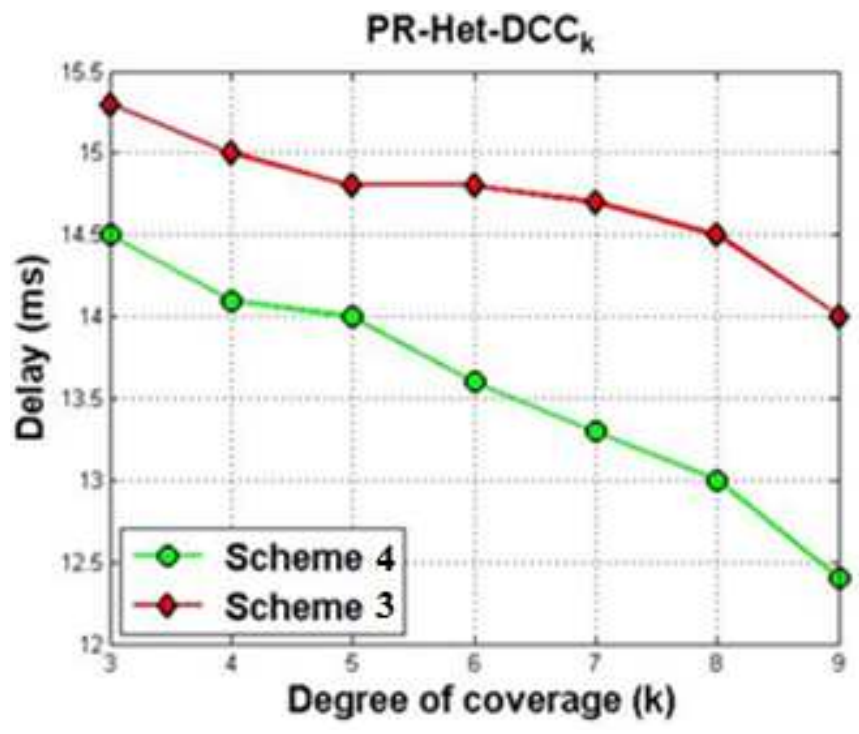

Figure 14: Comparing data gathering protocols (Delay)

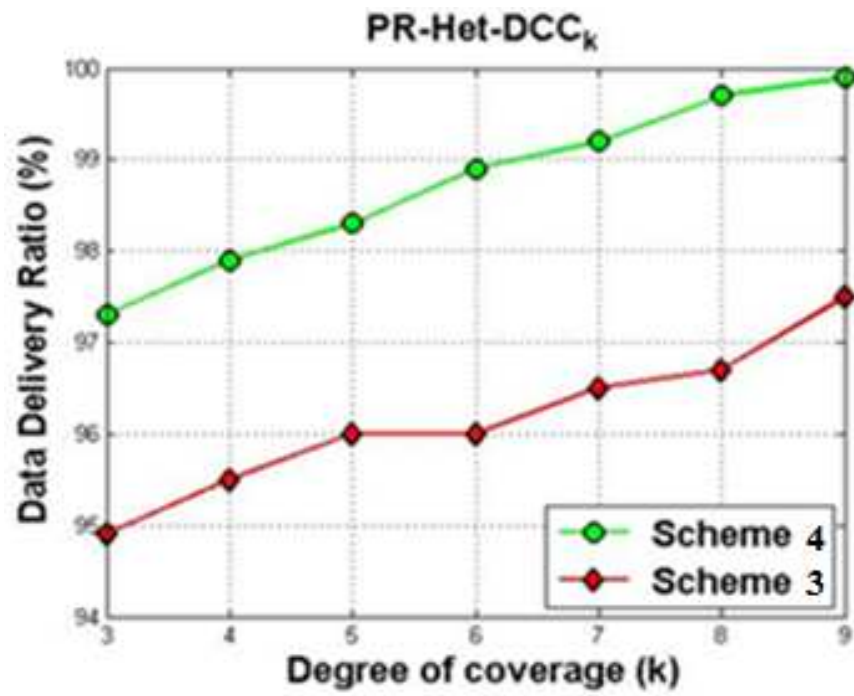

Figure 15: Comparing data gathering protocols (Data delivery ratio)

Now, we use our proposed data gathering protocol using adaptive hybrid forwarding (Scheme 3). Figure 12 shows that PR-Het-DCC $C_{k}$ incurs less delay than R-Hom-DCC ${ }_{k}$ as heterogeneous sensors tend to transmit data over long distances, thus, reducing the number of forwarders between source sensors and the mobile sink $m s_{1}$. This results in time saving due to less processing of the data before it reaches the sink. Also, as shown in Figure 13, PR-Het-DCC ${ }_{k}$ has better data delivery ratio than R-Hom- $\mathrm{DCC}_{k}$. This higher delay caused by R-Hom-DCC $\mathrm{C}_{k}$ may yield data loss since the next forwarder may not be active in the next round.

We compared between our proposed data gathering protocol using optimal sink mobility (Scheme 4) as stated in Section 6 with our adaptive hybrid data forwarding protocol (Scheme 3), which is discussed in Section 5. We considered our PR-Het-DCC $k$-coverage protocol. We found that Scheme 4 outperforms Scheme 3 in terms of average delay (Figure 14) and average data delivery ratio (Figure 15). Clearly, Scheme 4 provides more guarantee for delivering data to the mobile sink given its pattern of mobility. Also, Scheme 4 less delay than Scheme 3 for the above reason. This shows the positive impact of optimal sink mobility on the global system performance.

\section{Conclusion}

\subsection{Summary}

In this paper, we focused on the problem of joint $k$-coverage and data gathering in heterogeneous sensor nets using two different strategies. While the first data collection protocol uses adaptive hybrid forwarding, the second one is based on optimal sink mobility in a circular sensor field. First, we proposed centralized and distributed approaches to solve the connected $k$-coverage in this type of sensor nets, where the sensor field is decomposed into concentric circular bands. Each band contains the same type of sensors with regard to their initial energy reserve, sensing range, and communication range. However, each band has different type of sensors from all other bands with respect to the above characteristics. Second, we presented an adaptive hybrid forwarding-based data collection protocol, where the sink is immobile. Our data forwarding protocol using a hybrid scheme benefits from the advantages of deterministic and opportunistic forwarding schemes. Third, we investigated sink mobility in a circular sensor field, and computed the mobility trajectory of the sink that minimizes the total energy consumption due to data and control packet communication, as well as sink mobility. We derived a closed-form solution that characterizes the optimal mobility trajectory of the sink. Furthermore, we suggested a data collection protocol based on this optimal mobility of the sink. With regard to our simulation results, not surprisingly and conform to previous results on the impact of heterogeneity on the design of sensor nets [28], we found that our $k$-coverage protocols for heterogeneous sensor networks outperform their counterparts for homogeneous sensor nets. Moreover, we found that our data collection protocol using our proposed optimal sink mobility is more efficient in terms of average delay and 
average data delivery ratio compared to our adaptive hybrid geographic forwarding-based data collection protocol.

\subsection{Future Work}

Our future work is five-fold. In this work, we assumed that the sensors are densely deployed. First, we want to investigate the problem of joint $k$-coverage and data collection in sparse sensor networks, where it may not be always possible to $k$-cover the whole sensor deployment field at once. Second, we plan to extend our current study to stochastic $k$-coverage in heterogeneous sensor nets using a probabilistic sensing model. Particularly, we consider a non0-1 sensing model (i.e., not a sensing disk model), where the sensing capabilities of the sensors are not necessarily the same in all the directions. Third, we focus on the problem of joint $k$-coverage and data collection in three-dimensional heterogeneous mobile sensor nets [4], such as underwater sensor nets, by exploiting the concept of Reuleaux tetrahedron (i.e., a counterpart of Reuleaux triangle in threedimensional space) [34]. We expect the presence of three factors (i.e., heterogeneity, three-dimensional space, and mobility) to make the problem more challenging. Fourth, we plan to assess the performance of our joint protocols for $k$ coverage and data collection using a sensor testbed.

As it can be seen from the equation system shown in (2) and (3) above, our proposed hierarchical architecture has some limitation. The sensor deployment field cannot have more than a fixed number $n_{b}$ of concentric circular bands. The value of $n_{b}$ is determined based on the radius of the circular sensor field, $D$, and the minimum and maximum sensing capabilities of the sensors, namely $r_{\min }$ and $r_{\max }$, respectively. As our fifth future work, we plan to extend our current analysis to alleviate this problem, thus, allowing any number of concentric circular bands in the field. This will enable the deployment of various types of sensors with respect to their sensing capabilities, and without any restriction.

\section{Acknowledgments}

The author gratefully acknowledges the insightful comments of the anonymous reviewers which helped improve the quality and presentation of the paper significantly. This work is partially supported by the US National Science Foundation (NSF) grants CNS-0917089 and CNS-1054935.

\section{References}

[1] H. M. Ammari and S. K. Das, "Centralized and clustered $k$ coverage protocols for wireless sensor networks," IEEE Transactions on Computers, 61(1), pp. 118-133, Jan. 2012.

[2] H. M. Ammari and J. Giudici, "On the connected $k$-coverage problem in heterogeneous sensor nets: The curse of randomness and heterogeneity," Proc. IEEE International Conference on Distributed Computing Systems (ICDCS), pp. 265-272, 2009.
[3] H. M. Ammari, "Investigating the energy sink-hole problem in connected $k$-covered wireless sensor networks," IEEE Transactions on Computers, 63(11), pp. 2729-2742, Nov. 2014.

[4] H. M. Ammari, "On the problem of $k$-coverage in 3D wireless sensor networks: A Reuleaux tetrahedron-based approach," Proc. IEEE International Conference on Intelligent Sensors, Sensor Networks and Information Processing (ISSNIP), pp. 1-8, 2011.

[5] X. Bai, S. Kumar, D. Xuan, Z. Yun, and T. Lai, "Deploying wireless sensors to achieve both coverage and connectivity," Proc. ACM Symposium on Mobile Ad Hoc Networking and Computing (MobiHoc), pp. 131-142, 2006.

[6] S. Biswas and R. Morris, "Opportunistic routing in multihop wireless networks," ACM SIGCOMM Computer Communication Review, 34(1), pp. 69-74, 2004.

[7] B. Bollobás, The Art of Mathematics: Coffee Time in Memphis, Cambridge University Press, 2006.

[8] N. Bulusu, J. Heidemann, and D. Estrin, "GPS-less low cost outdoor localization for very small devices," IEEE Pers. Communications Magazine, 7(5), pp. 28-34, 2000.

[9] B. Chen, K. Jamieson, H. Balakrishnan, and R. Morris, "Span: An Energy-Efficient Coordination Algorithm for Topology Maintenance in Ad Hoc Wireless Networks," Proc. ACM Annual International Conference on Mobile Computing and Networking (MobiCom), pp. 85-96, 2001.

[10] X. Du and F. Lin, "Maintaining differentiated coverage in heterogeneous sensor networks," EURASIP Journal on Wireless Comm. and Net., 5(4), pp. 565-572, 2005.

[11] E. J. Duarte-Melo and M. Liu, "Analysis of energy consumption and lifetime of heterogeneous wireless sensor networks," Proc. IEEE Global Communications Conference (Globecom), pp. 21-25, 2002.

[12] H. Gupta, Z. Zhou, S. Das, and Q. Gu, "Connected sensor cover: Self-organization of sensor networks for efficient query execution," IEEE/ACM Transactions on Networking, 14(1), pp. 55-67, 2006.

[13] C. Huang, Y. Tseng, and H. Wu, "Distributed protocols for ensuring both coverage and connectivity of a wireless sensor network," ACM Transactions on Sensor Networks, 3(1), pp. 1-24, 2007.

[14] B. Karp and H. T. Kung, "GPSR: Greedy perimeter stateless routing for wireless networks," Proc. ACM Annual International Conference on Mobile Computing and Networking (MobiCom), pp. 243-254, 2000.

[15] L. A. Klein, "A boolean algebra approach to multiple sensor voting fusion," IEEE Trans. Aerospace and Electronic Systems, 29(2), pp. 317-327, Apr. 1993.

[16] L. Lazos, R. Poovendran, and J. Ritcey, "On the deployment of heterogeneous sensor networks for detection of mobile targets," Proc. International Symposium on Modeling and Optimization in Mobile, Ad Hoc, and Wireless Networks (WiOpt), pp. 1-10, 2007.

[17] J. Luo and J.-P. Hubaux, "Joint mobility and routing for lifetime elongation in wireless sensor networks," Proc. IEEE Conference on Computer Communications (INFOCOM), pp. 1735-1746, 2005. 
[18] V. P. Mhatre, C. Rosenberg, D. Kofman, R. Mazumdar, and N. Shroff, "A minimum cost heterogeneous sensor network with a lifetime constraint," IEEE TMC, 4(1), pp. 415, Jan.-Feb. 2005.

[19] Y. Ma, S. Dalal, M. Alwan, and J. Aylor, "ROP: A resource oriented protocol for heterogeneous sensor networks," Proc. Virginia Tech's Symposium on Wireless Personal Communication, pp. 59-70, 2003.

[20] F. G. Nakamura, F. P. Quintão, G. C. Menezes, and G. R. Mateus, "An optimal node scheduling for flat wireless sensor networks," Proc. International Conference on Networking (ICN), LNCS, 3420, pp. 475-482, 2005.

[21] D. Nicules and B. Nath, "Ad-hoc positioning system using AoA," Proc. IEEE Infocom, 2003, pp. 1734-1743.

[22] T. Sun, L. Chen, C. Han, and M. Gerla, "Reliable sensor networks for planet exploration," Proc. IEEE Conf. Networking, Sensing and Control, 2005, pp. 816-821.

[23] M. M. Vieira, L. M. Vieira, L. B. Ruiz, A. F. Loureiro, A. O. Fernandes, and J. S. Nogueira, "Scheduling nodes in wireless sensor networks: A Voronoi approach," Proc. IEEE International Conference on Local Computer Networks $(L C N), 2003$.

[24] Y.-C. Wang and Y.-C. Tseng, "Distributed deployment schemes for mobile wireless sensor networks to ensure multi-level coverage," IEEE Transactions on Parallel and Dist. Systems, 19(9), pp. 1280-1294, Sep. 2008.

[25] Y. Wang, X. Wang, D. P. Agrawal, and A. A. Minai, "Impact of heterogeneity on coverage and broadcast reachability in wireless sensor networks," Proc. IEEE International Conference on Computer Communications and Networks (ICCCN), pp. 63-67, 2006.

[26] G. Xing, X. Wang, Y. Zhang, C. Lu, R. Pless, and C. Gill, "Integrated coverage and connectivity configuration for energy conservation in sensor networks," ACM Transactions on Sensor Networks, 1(1), pp. 36-72, 2005.

[27] S. Yang, F. Dai, M. Cardei, and J. Wu, "On connected multiple point coverage in wireless sensor networks," Int. Journal of Wireless Information Networks, 13(4), pp. 289301, 2006.

[28] M. Yarvis, N. Kushalnagar, H. Singh, A. Rangarajan, Y. Liu, and S. Singh, "Exploiting heterogeneity in sensor networks," Proc. IEEE Conference on Computer Communications (INFOCOM), pp. 878-890, 2005.

[29] F. Ye, G. Zhong, J. Cheng, S. Lu, and L. Zhang, "PEAS: A robust energy conserving protocol for long-lived sensor networks," Proc. IEEE International Conference on Distributed Computing Systems (ICDCS), pp. 1-10, 2003.

[30] M. Yuce, P. Ng, C. Lee, J. Khan, and W. Liu, "A wireless medical monitoring over a heterogeneous sensor network," Proc. IEEE International Conference of the IEEE Engineering in Medicine and Biology Society (EMBS), pp. 5894-5898, 2007.

[31] H. Zhang and J. Hou, "Maintaining sensing coverage and connectivity in large sensor networks," Ad Hoc \& Sensor Wireless Networks, 1(1-2), pp. 89-124, 2005.

[32] Y. Zou and K. Chakrabarty, "A distributed coverage- and connectivity-centric technique for selecting active nodes in wireless sensor networks," IEEE Transactions on Computers, 54(8), pp. 978-991, 2005.

[33] mathworld.wolfram.com/ReuleauxTriangle.html.

[34] mathworld.wolfram.com/ReuleauxTetrahedron.html 